\title{
The SHiP experiment at CERN
}

\author{
Antonio luliano*t \\ Università di Napoli Federico II and INFN \\ E-mail: antonio.iulianodcern.ch
}

Research for new physics is currently one of the main tasks of particle physics, looking for new particles having high masses (energy frontier) or very feebly interacting (intensity frontier). The SHiP experiment has been proposed in 2015 to search for hidden particles at the intensity frontier. It is designed to be located in a new beam dump facility, receiving $400 \mathrm{GeV}$ protons from the CERN SPS accelerator. A total of $2 \times 10^{20}$ protons on target is expected to be collected in five years of data taking. The experiment plans to explore a wide domains of couplings and masses for light long-lived exotic particles. Their decays in a $50 \mathrm{~m}$ long vacuum vessel can be reconstructed in a background free environment, allowing for a high sensitivity of the experiment. Moreover, the experiment is suitable to reconstruct tau neutrino interactions, with expected yields orders of magnitude larger than previous experiments. This provides interesting possibilities of study such as the first observation of $\bar{v}_{\tau}$, the measurement of $v_{\tau}$ and $\bar{v}_{\tau}$ cross-sections with high statistics and the exploration of the charm content of the nucleon. Ancillary measurements to validate the Monte Carlo predictions have been performed in July 2018, and their analysis is currently ongoing.

XXVII International Workshop on Deep-Inelastic Scattering and Related Subjects - DIS2019

8-12 April, 2019

Torino, Italy

\footnotetext{
${ }^{*}$ Speaker.

${ }^{\dagger}$ On Behalf of the SHiP Collaboration
} 


\section{Introduction}

The Standard Model provides a clear and accurate description of the elementary constituents of matter. However, this model cannot be considered a complete theory, as it fails to describe several well-established observed phenomena such as the existence of dark matter, the baryon asymmetry in the universe and the small values of neutrino masses. Therefore, many Beyond Standard Model (BSM) theories have been proposed, to solve these puzzles by adding new particles to the currently known ones.

The research for new particles is one of the main topics of contemporary particle physics, using data both from accelerators and from astronomical sources. However, no clear evidence has been found so far, yet still a vast region of masses and couplings remains unexplored. The high energy frontier consists in the research for new phenomena at high energies, such as the production of new high mass particles, while the intensity frontier is defined by the assumption of very weakly interacting particles, with average mass, $\mathscr{O}(\mathrm{GeV})$.

In this landscape, the Search for Hidden Particles (SHiP) experiment plans to explore a large parameter region at the intensity frontier, in a beam dump facility at the CERN SPS accelerator.

The experiment has been proposed in 2015, with a submission of a Technical Proposal [1] and a Physics Paper [2]. Since 2016 it has been part of the Physics Beyond Colliders research group [3] and it is currently in the design phase [4].

\section{General structure of the experiment}

The SHiP experiment looks for new long lived very weakly interacting particles, also known as Hidden Particles, predicted by various Beyond the Standard Model theories. These particles can be produced from the interactions of $400 \mathrm{GeV}$ protons, impinging on a fixed target. The experiment will be located at the North Area of the CERN SPS accelerator, in a new beam dump facility which will provide various research possibilities. A total of $2 \times 10^{20}$ protons on target are expected to be collected, during a data taking period of 5 years.

The overall structure of the SHiP experiment is shown in Figure 1. In order to maximise the sensitivity of the experiment, the production of Hidden Particles from the decays of heavy hadrons should be enhanced, but at the same time it is fundamental to make all the background from Standard Model negligible.

The composition and length of the proton target have been designed to maximise the production of charm and beauty, and to enchance the absorbtion of kaons and pions before their decays. The designed target presents an hybrid structure, which consists of blocks of titanium-zirconium doped molybdenum alloy (TZM) in the core of the shower, followed by blocks of pure tungsten. The total target length is $150 \mathrm{~cm}$, covering 12 interaction lengths.

The proton target is followed by a $5 \mathrm{~m}$ iron hadron stopper, dedicated to the containment of the produced hadron shower. On the other hand, a large fraction of the produced muons is not absorbed. Therefore, a dedicated magnetic muon shield has been designed, aimed to deflect muons out of the acceptance of downstream detectors. Thanks to the magnetization of the last section of the hadron absorber, it is possible to constrain the muon shield length to $35 \mathrm{~m}$, at the same time reducing the muon flux of four orders of magnitude [5]. 


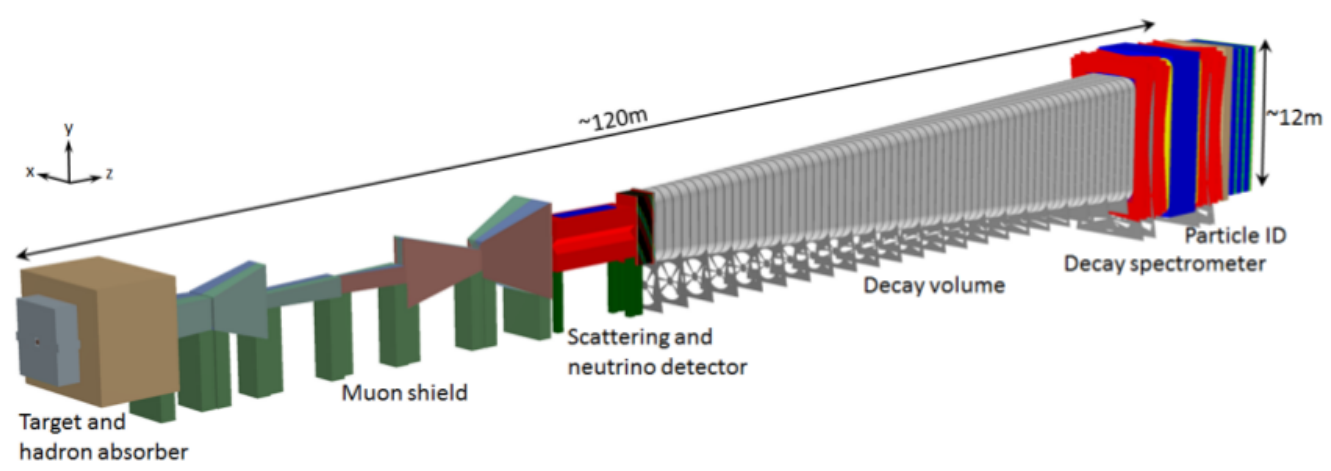

Figure 1: Overall layout of the SHiP experiment, as presented in the SHiP CDS progress report [4].

A $50 \mathrm{~m}$ long decay vessel is used to locate the decay of Hidden Sector particles. It is maintained at a pressure of $10^{-3}$ bar in order to minimize the background from neutrino interactions. The decay vessel is followed by a spectrometer with straw trackers, dedicated to the reconstruction of the decay vertices. The most downstream part of the experiment is made of calorimeters and a muon system, providing full information of the event.

\section{The Scattering and Neutrino Detector}

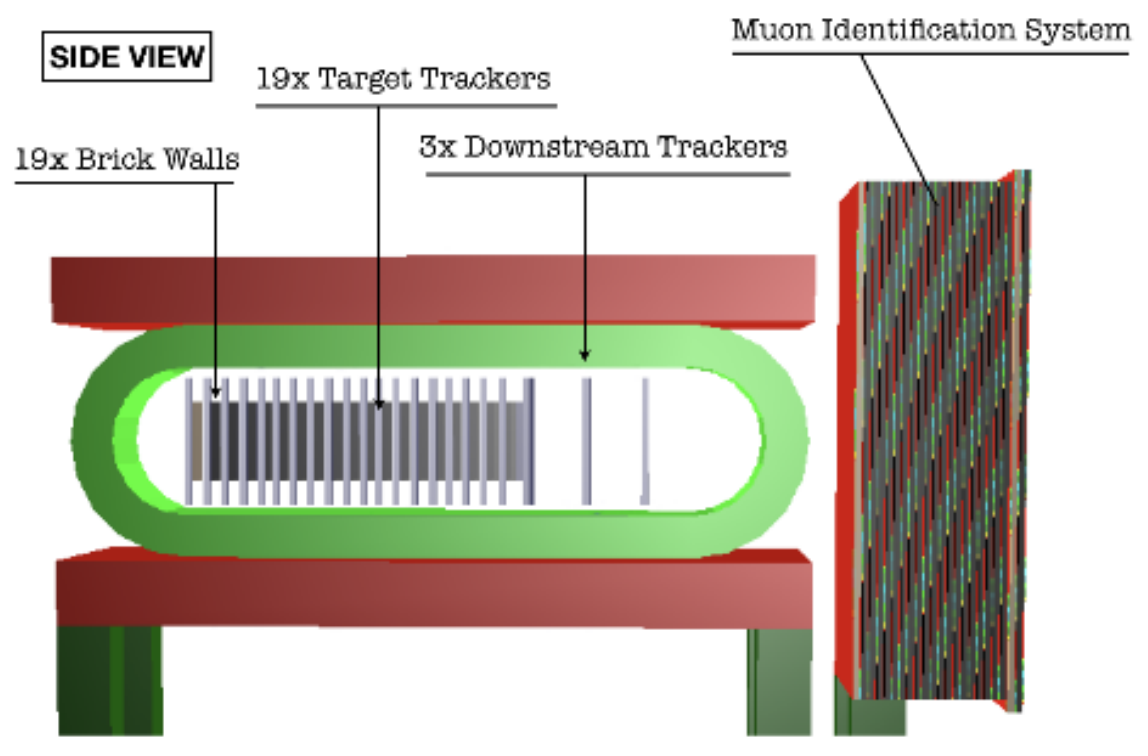

Figure 2: Layout of the Scattering and Neutrino detector. The two main components, the magnetized detector and the muon filter, are visible.

The structure of the SHiP experiment allows the production of a high intensity neutrino beam in a clean environment, thanks to the absorption of hadrons and the deflection of muons from the 
proton target. Therefore, a neutrino detector has been designed between the magnetic muon shield and the decay vacuum vessel. At the same time, the detector is suitable for Light Dark Matter search, by looking for an isolated electromagnetic shower produced by the recoil electron.

The layout of the designed detector is shown in Figure 2. The two main components are a magnetized detector, where neutrino interactions can be reconstructed in an emulsion/lead target, and a muon filter.

The emulsion detector is a modular target, where the unit cell is the Emulsion Cloud Chamber (ECC), made of lead plates interleaved with nuclear emulsion films, acting as the main target for the neutrino interactions. Each ECC is followed by a Compact Emulsion Spectrometer, where three emulsions separated by air gaps are used to measure the particle curvature in a magnetic field, thus providing the charge and momentum of the charged particles produced in the neutrino interactions. The cells with emulsions are organized in walls, interleaved with electronic Target Trackers to provide the time information for each event. Other trackers are placed downstream of the target, in order to improve momentum resolution for penetrating particles and to match them with the following muon filter.

The muon filter is made of RPC tracker stations, interleaved with iron slabs. It provides muon identification for the neutrino detector, and at the same time it is able to tag neutrino interactions in the passive material surrounding the detectors. Tagging these interactions allows the identification of produced long-lived neutral particles decaying in the downstream vacuum vessel, which could mimick signals of Beyond the Standard Model physics.

\section{Expected performance of the Scattering and Neutrino Detector}

The estimated neutrino fluxes produced in the beam dump during the five years of data taking are reported in Table 1, along with the expected number of charged current Deep Inelastic Scattering (CC-DIS) events. Tau neutrinos are the flavour of larger interest, because currently there

\begin{tabular}{c|c|c} 
& Beam dump & CC DIS interactions \\
\hline$N_{v_{e}}$ & $2.8 \times 10^{17}$ & $1.1 \times 10^{6}$ \\
$N_{v_{\mu}}$ & $4.2 \times 10^{18}$ & $2.7 \times 10^{6}$ \\
$N_{v_{\tau}}$ & $1.4 \times 10^{16}$ & $3.2 \times 10^{4}$ \\
$N_{\bar{v}_{e}}$ & $2.3 \times 10^{17}$ & $2.6 \times 10^{5}$ \\
$N_{\bar{v}_{\mu}}$ & $2.7 \times 10^{18}$ & $6.0 \times 10^{5}$ \\
$N_{\bar{v}_{\tau}}$ & $1.4 \times 10^{16}$ & $2.1 \times 10^{4}$ \\
\hline
\end{tabular}

Table 1: Expected neutrino flux for different neutrino flavours after collecting $2 \times 10^{20}$ protons on target.

are only few measurements available. At the energies of the SHiP experiment, the main source is the decay of a $D_{s}$ meson. A pair of tau neutrinos is produced from each $D_{s}$, one in the prompt $D_{s} \rightarrow \tau \nu_{\tau}$ decay and the other one in the subsequent decay of the $\tau$ lepton. The usage of both high resolution spatial detectors and a magnetic field allows to locate production and decay vertices, and to measure the charge of the associated $\tau$ lepton in the hadronic and in the muonic decay channels.

The detection of a high number of tau neutrino interactions will allow a detailed study of the interaction cross sections. The larger mass of the associated $\tau$ lepton, with respect to the muon 
and electron flavours, allows in fact to scope the $F_{4}$ and $F_{5}$ structure functions in the CC DIS cross section:

$$
\begin{aligned}
\frac{d^{2} \sigma^{v(\bar{v})}}{d x d y}=\frac{G_{F}^{2} M E_{v}}{\pi\left(1+Q^{2} / M_{W}^{2}\right)^{2}}( & \left(y^{2} x+\frac{m_{\tau}^{2} y}{2 E_{v} M}\right) F_{1}+\left[\left(1-\frac{m_{\tau}^{2}}{4 E_{v}^{2}}\right)-\left(1+\frac{M_{x}}{2 E_{v}}\right)\right] F_{2}+ \\
& \left. \pm\left[x y\left(1-\frac{y}{2}\right)-\frac{m_{\tau}^{2} y}{4 E_{v} M}\right] F_{3}+\frac{m_{\tau}^{2}\left(m_{\tau}^{2}+Q^{2}\right)}{4 E_{v}^{2} M^{2} x} F_{4}-\frac{m_{\tau}^{2}}{E_{v} M} F_{5}\right),
\end{aligned}
$$

where the plus sign applies to neutrino scattering, and the minus one to antineutrino scattering. $M$ and $m_{\tau}$ are the nucleon and $\tau$ lepton masses, respectively, $M_{W}$ is the $\mathrm{W}$ boson mass and $E_{v}$ is the initial neutrino energy.

An other important field of neutrino physics is the neutrino-induced charm production, with more than $\sim 2 \times 10^{5}$ charmed hadrons expected. Several studies on charm physics will be performed, such as the double charm production cross sections and the search for pentaquarks with charm contents. Moreover, charm production in neutrino scattering is highly sensitive to the strange quark content of the nucleon, especially in the case from antineutrinos interactions, where the squark is dominant. SHiP will improve significantly the uncertainty on the strange quark distribution in the nucleon.

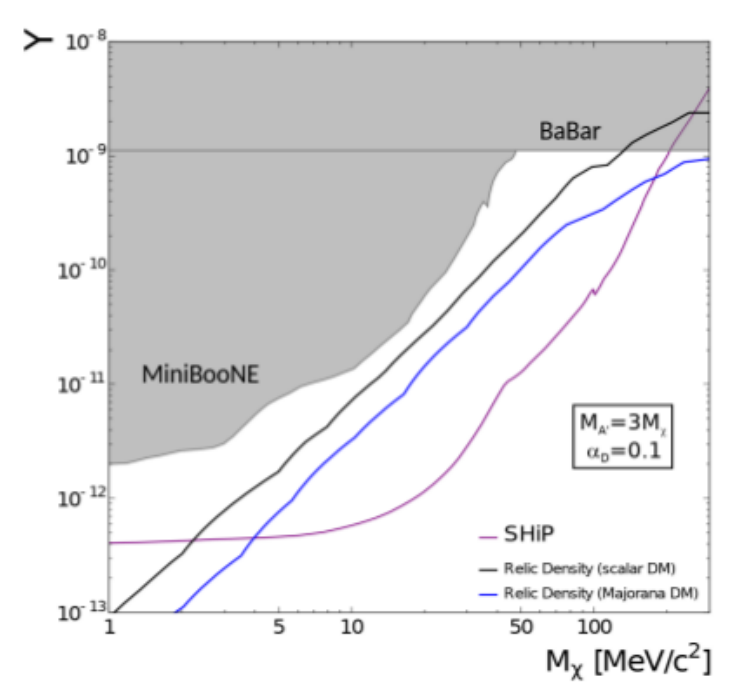

Figure 3: Estimated sensitivity of the SHiP experiment to Light Dark Matter scattering.

Light Dark Matter scattering in the emulsion detector produces an isolated electromagnetic shower, originated from the recoil electron. This shower can be then detected in the Emulsion Cloud Chamber, working as a fine sampling electromagnetic calorimeter. Background from neutrino interactions can be tagged by identification of the production vertex or by the detection of additional visible energy along the electron. Finally, kinematics correlation between the energy and the azimuthal angle of the scattered electron can be used. The estimated sensitivity is shown in Figure 3. 


\section{Ancillary measurements}

In order to validate the Monte Carlo simulations used in the design of the SHiP experiment, the muon flux and the charmed hadrons produced have been studied with two different experimental configurations, exposed to a $400 \mathrm{GeV}$ beam from the SPS accelerator. The exposures have been performed in three weeks at July 2018, in the H4 beam line.

The configuration for the muon flux measurement presented a $10 \times 154.3 \mathrm{~cm}^{3}$ replica of SHiP target, followed by a $2.4 \mathrm{~m}$ hadron absorber. The muon momentum spectrum can be measured by a spectrometer consisting of drift tubes stations installed upstream and downstream of the Goliath magnet. The last detector downstream is a muon tracking system made of five RPC stations. The total number of protons collected in the target amounted to $6 \times 10^{11}$.

The configuration for measuring the total charm production yield is shown in figure 4 . The muon flux setup has been modified, by keeping the Goliath magnet and the RPC stations, but replacing the SHiP replica target with a lead target instrumented with nuclear emulsion films. Upstream of the target a pixel detector has been installed, to cope with the higher multiplicity of tracks. Drift tubes and SciFi detectors have been used as spectrometer stations downstream of the Goliath magnets. The total number of protons collected in the target amounted to $1.6 \times 10^{6}$.

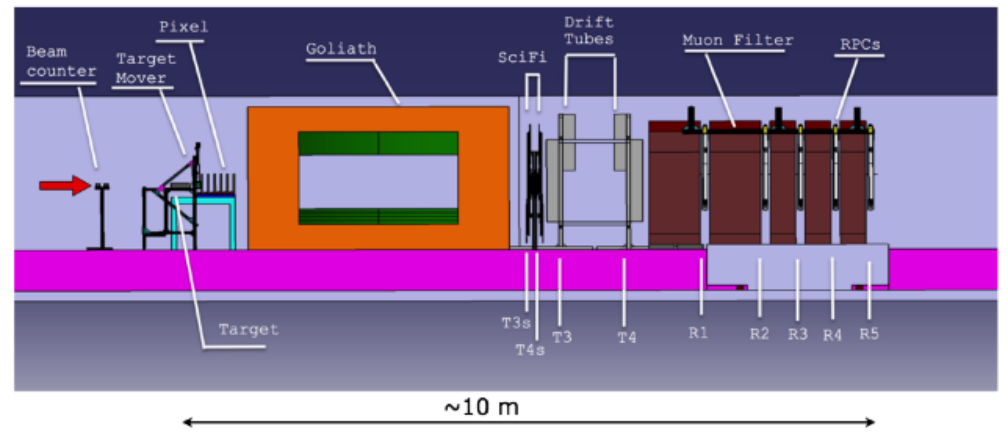

Figure 4: Layout of the apparatus used to study the charm production yield in July 2018.

Analysis of the data collected in the two configurations is currently ongoing. The comparison of the results with the Monte Carlo predictions will provide in the next years important information for the optimization of the design of the SHiP experiment.

\section{References}

[1] SHiP Collaboration, M.Anelli et al., A Facility to Search for Hidden Particles (SHiP) at the CERN SPS, CERN-SPSC-2015-016, SPSC-P-350, 8 April 2015 [arXiv:1504.04956].

[2] S. Alekhin et al., A facility to Search for Hidden Particles at the CERN SPS: the SHiP physics case Rept. Prog. Phys. 79 (2016), no. 12124201 [arXiv: 1504.04855$].$

[3] Physics Beyond Colliders Study Group, R. Alemany et al., Summary Report of Physics Beyond Colliders at CERN, CERN-PBC-REPORT-2018-003 [arXiv:1902.00260].

[4] SHiP Collaboration, SHiP Experiment, Progress Report, CERN-SPSC-2019-010 / SPSC-SR-248

[5] SHiP Collaboration, The active muon shield in the SHiP experiment, JINST 12 (2017) P05011 [arXiv:1703.03612]. 